\title{
Projecting two words with one machine: Presenting two different visual stimuli using just one projector without viewers' noticing the duality
}

\author{
Kazuo Mori \\ Shinshu University, Tokyo, Japan
}

\begin{abstract}
A new method, named the fMORI technique, for presenting two different visual stimuli using just one video projector without the viewers' noticing the duality is introduced along with several sample applications of this presentation method. The LCD projectors available now have three color LCD panels aligned differently in terms of their polarization, polarizing differently the green and magenta (red plus blue) components of its emitting image. Therefore, two visual stimuli either in green or in magenta projected on the same screen can be observed separately by subjects wearing an appropriate polarizing filter. This is a variation of the MORI technique (Mori, 2003, 2007b) but it requires only one video projector rather than two. Full-color movies cannot be presented with the fMORI technique, but it may be utilized in various research areas in which only static monochrome visual stimuli, such as words or numbers, are used.
\end{abstract}

The MORI technique reported in Mori (2003), which used two LCD video projectors, would not work with new LCD type projectors because their alignment of LCD panels has been changed so as to be crossed, whereas previously they were aligned in the same way in terms of polarization. In the new type, the LCD panel for green is placed perpendicular to the other two LCD panels for red and blue. Thus, a single polarizer cannot block the full-color image emitted from the new LCD projectors. It blocks either green or magenta but fails to shut out the other fraction. Since the basic principle of the MORI technique is to let two groups of viewers to observe two different images separately by wearing suitable polarizing sunglasses that either transmit or block one of the two images, being unable to shut out the image with a single polarizer is a crucial problem.

Mori (2007b) provided a solution for this problem by utilizing DLP projectors instead of LCD ones. The DLP projectors emit a light beam without polarizing it. Therefore, by placing an appropriate polarizing filter in front of each of the two DLP projectors, the experimenter can create two different channels to present two images polarized differently to each other, as described in Mori (2003).

Another solution to the LCD alignment problem is presented in this paper. Since the green and magenta (red plus blue) fractions of light can be blocked separately, these two fractions can be used to convey two different images separately. In this way, a single LCD projector can present two different images, either in green or magenta, which can be seen separately by two groups of viewers wearing polarizing sunglasses which allow them to view only one of the two images.
In the present article, this much simpler way to utilize the MORI technique will be described in detail along with sample applications that have been conducted in a variety of research areas in the behavioral sciences.

\section{THE IMORI TECHNIQUE}

The new MORI technique, named fMORI, by turning the problem caused by the mixed alignment of LCD projectors into a merit, has a considerable advantage over both the original (Mori, 2003) and the revised one (Mori, $2007 b$ ) in that it requires just one projector. It can project two words with one machine. Although it utilizes the polarizing property of light using the same basic principle as the original MORI technique and the revised one, the two channels are built-in within one LCD projector thanks to the different alignment of the three LCD panels for each of the three primary colors, red, green, and blue.

\section{Basic Principle}

The basic principle of the fMORI technique is the same as the former ones (Mori, 2003, 2007b). It employs the polarization properties of light, which have been used in certain types of 3-D displays. In a 3-D presentation, two different images are seen separately by two eyes, with the right and left eyes wearing a different type of polarizing filter. In the MORI technique, two different images are seen by two different persons with different types of polarizing sunglasses. The crucial merit of the MORI technique is that viewers are to observe two different images without their noticing the duality since the polarization cannot be detected by the human eye. 
In the fMORI technique, two different images presented on the same screen, one in green and the other in magenta, can be seen separately by two groups of viewers. Participants are asked to wear a pair of polarizing sunglasses which look similar to ordinary ones, but can filter out one of the projected images. The simplicity of the experimental setting should help prevent the participants from becoming suspicious about the presentation trick. The point is that the participants should think they are observing the same stimulus together when in fact they are observing two different images (see Figure 1).

\section{Apparatus}

Video projector. The fMORI technique requires just one LCD projector. Unlike the system for the original MORI technique (Mori, 2003), the old type of LCD projector is not suitable for the fMORI technique because their LCD panels are aligned in the same direction in terms of polarization. It can be ascertained whether a projector is the new type or not by placing a polarizing filter in front of the projection lens and turning it around its projection axis. If the light is blocked completely in a certain direction, the projector is an old type. If the color of the light changes, from greenish to pinkish or the other way around, it is the new type and is suitable for the fMORI technique.

Polarizing filters. Since the light beam emitted from the LCD projectors is already polarized in two ways depending on the color fractions, green and magenta, no additional polarizing filters are needed.

Polarizing sunglasses. The same type of linear polarizing sunglasses as used for the MORI technique is suitable for the fMORI technique. With these sunglasses, one can see the projected light as either greenish or pinkish depending on the type of sunglasses worn. The polarizing sunglasses can be made using ordinary sunglass frames and cutouts from a sheet of polarizing filter. Plastic sheets of linear polarizing filter are sold for use as school teaching materials or for 3-D presentation equipment. It costs about $¥ 6,000$, or US $\$ 50$ for a sheet of polarizing filter measuring $25 \mathrm{~cm} \times 25 \mathrm{~cm} \times 0.8 \mathrm{~mm}$, which can be cut easily into any size or shape using ordinary scissors.

Preparation of visual stimuli. The two different images to be projected by an LCD projector should be prepared as a single frame by combining two images drawn in either green or magenta. There are two options for presentation of images in the fMORI technique: black figures on either a green or magenta background or green or magenta figures on a black background. The preparation procedure for the former is described here because it has been applied to several experiments already, as will be described in the sample application section later.

The preparation of visual stimuli can be done with digital image processing software such as Adobe Photoshop or Photoshop Elements. The two images should be drawn separately on two layers each either in green or magenta. It is worth noting that when viewers wear green-passing sunglasses, figures drawn in magenta will be seen in black on a green background while figures drawn in green will be hidden in the background, and vice versa when viewers wear magenta-passing sunglasses. The researcher must be careful to use a pure green for drawing a figure in green, and to use a pure mixture of red and blue for drawing other figures in magenta. Digital image processing software can show the three primary color components separately and adjust each of them. Set 0 in the red and blue components for pure green, and 0 in the green component for pure magenta.

After having drawn the two images, the two layers are integrated with the logical conjunction principle. If the two images are drawn in green and magenta on a white background, the integrated image looks like two overlapping images with the intersections in black (see Figure 2).

The human eye is good at detecting lines. Therefore, the to-be-hidden images may not be hidden completely even if the integrated pictures are properly made in pure green or magenta. It is necessary to add some random noise after having integrated the two images so that the lines of the to-be-hidden image become obscured (see Figure 3). The noise addition is easily done using digital image processing software. It is also advisable to adjust the background colors in pale magenta rather than in plain white. The blockage of the green and magenta fractions of the light beam is not equal, since magenta is a mixture of red and blue while green has only a single component. Whether a to-be-hidden image can be covered successfully or not may depend on other varying factors, such as the shape of the image, the viewing conditions, etc.

Half-transparent screen. It is recommended that researchers use a half-transparent screen and project from

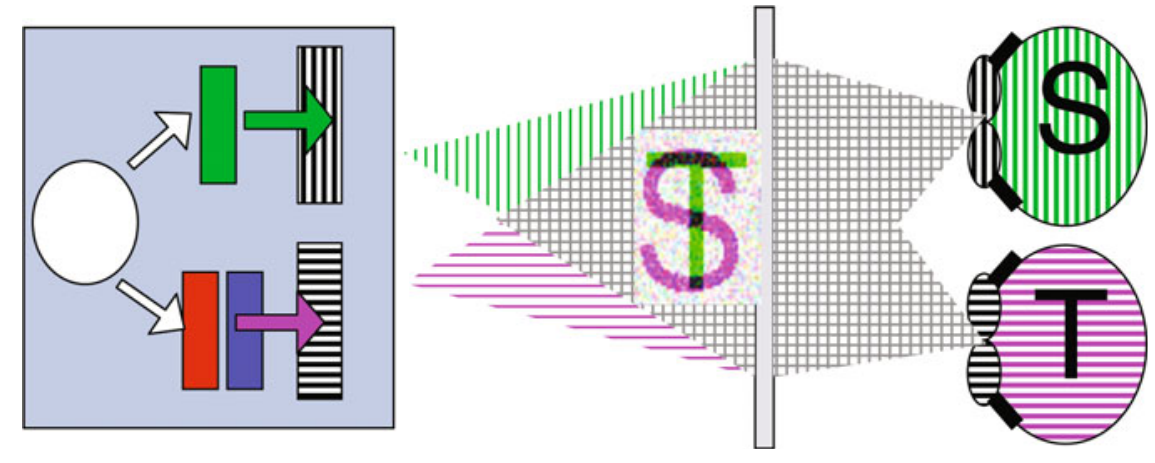

Figure 1. The two invisible channels created in the fMORI technique. 


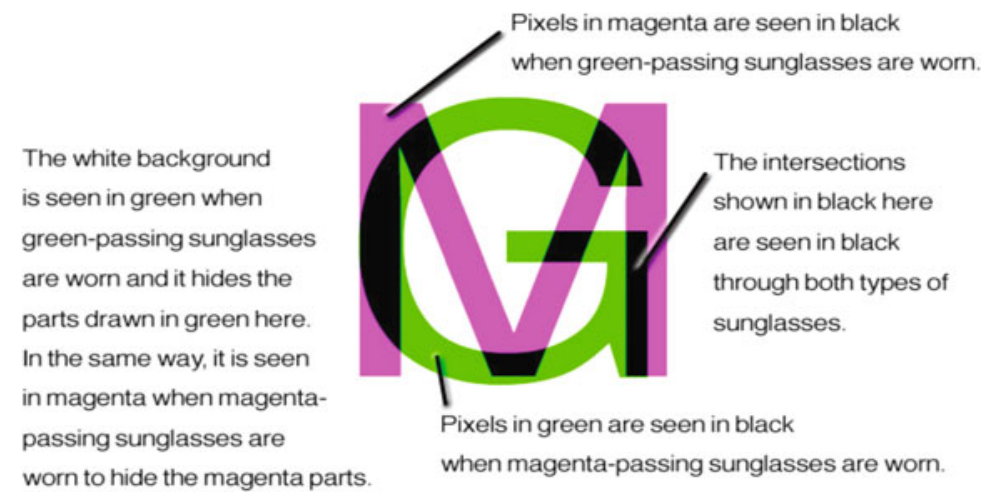

Figure 2. Integration of two images in green and magenta.

the rear. This is because ordinary screens tend to depolarize the polarized light beam at the point of reflection whereas half-transparent screens let the polarized beam pass through relatively unchanged. The rear projection causes screen glare. However, this provides a good excuse for having viewers wear sunglasses. If an ordinary projection system is employed, it is preferable to use the type of silver-coated screen that is used for 3-D movies.

\section{Some Tips for Practical Application}

Cover story for wearing sunglasses. Because of the rear projection, there is glare in the eyes of the viewers. Therefore, it is quite natural to tell them to wear the sunglasses to prevent the glare. There are several other possible ways to get the participants to wear sunglasses without having them become suspicious about it. In a former application of the MORI technique (Kanematsu, Mori, \& Mori, 1996/2003), the participants were told to wear sunglasses to simulate witnessing the event at night. In a recent study (Garry, French, Kinzett, \& Mori, in press), the participants were told they would be testing several levels of visual acuity by using sunglasses.

Prevention of detection of the trick. As long as the visual stimuli are well prepared, even very cautious viewers are unlikely to notice the duality. The successful reproduction of the Asch line judgment experiments to be reported below proved this. The participants could not detect the duality even when confronted with a discrepancy between the judgments of other participants and what they actually observed. However, one important point is to instruct the participants to keep their heads still, because if they tilt the head the polarization blockage can easily break down (cf. Mori, 2003). The simple instruction not to move the head has been found to be effective. If the experimenter tells them this instruction along with the others, the participants usually simply comply.

Collation of dual presentation without detection. To assure that the presentation trick has worked as the experimenters intended, it is recommended that there be an interview with the participants after the experiment to ask them whether they have noticed any duality in the visual stimuli. This could be done by means of a simple questionnaire. However, it is not advisable to ask them directly whether they have noticed the trick or not. Participants may answer "yes" to this question even though they have not noticed it because of vanity. In order to avoid such vanity self-reports, it is desirable to ask them whether they noticed any abnormality during the presentation period.

\section{Presentation of Easy and Difficult Anagram Tasks Using the fMORI Technique}

Several experiments have already been carried out utilizing the fMORI technique that have confirmed its effectiveness (Mori, 2005; Mori \& Arai, 2007; Mori \& Uchida, 2007). The first attempt (Mori, 2007a) to utilize the fMORI technique for psychological research was a replication of Alicke, LoSchiavo, Zerbst, and Zhang (1997) without using

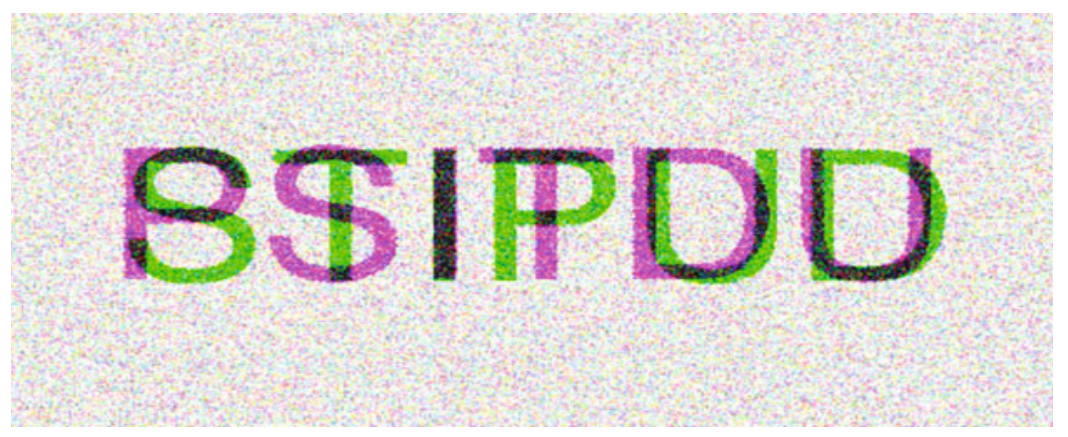

Figure 3. Overlapped images of two anagrams with random noise. In the actual experiment, anagram tasks were given in the Japanese language. 
confederates. Alicke et al. (1997) had pairs of participants (one of them a confederate) take a kind of perceptual intelligence test together. The participants were either to outperform the confederate or be outperformed and afterward evaluate the intellectual ability of their counterparts (confederates). For each pair of contestants (a naive participant and a confederate), another naive participant observed their performance and evaluated their intellectual ability. As Alicke et al. anticipated, those outperformed tended to exaggerate the ability of the outperformers in order to protect their self-images against unfavorable social comparisons. The outperformed participants evaluated their counterparts (confederates) much more highly than observer/participants did. Alicke et al. named this tendency to exaggerate an outperformer's ability the "genius effect."

In Mori (2007a), ten mutually acquainted foursomes of undergraduates participated in social comparison experiments similar to those of Alicke et al. (1997). However, there were no confederates needed when researchers used the fMORI technique which allowed presentation of two different anagram tasks surreptitiously to two groups of participants so that the one given an easier task could outperform the other (see Figure 3).

In each foursome, pairs were randomly assigned to be either performers or observers. For each pair, either easier anagram tasks or more difficult ones were presented with subjects unaware of the disparity in the tasks. Postexperimental interviews revealed that no participants noticed the presentation deceit. The performers who were presented easier tasks outperformed their counterparts in all ten groups. Those who were outperformed tended to evaluate their own intellectual levels as lower than how the observers evaluated them and evaluated their "opponents" the same as the observers did. Therefore, no "genius effect" was observed in Mori (2007a).

Mori and Uchida (2007) applied the fMORI technique to an educational study. Twenty-four junior high school students (4 in each of six classes) with academic achievement in the 26-50 percentiles were given easier anagram tasks while their 183 classmates were given difficult ones by means of the fMORI technique. The two series of anagram tasks were projected simultaneously on the same screen, but two groups of students wearing polarizing sunglasses observed only one series, unaware of the presence of the other. The target students given easier tasks performed significantly better than their classmates who served as the controls (see Figure 4).

Although it was a contrived success, the students' successful performance and subsequent social approval in class significantly promoted their sense of self-efficacy on the anagram tasks. The elevated sense of self-efficacy persisted as long as two years after the anagram task success. Moreover, a statistically significant number of the target students (17 out of 24) raised their academic achievement results for as long as 6 months after their contrived success on the anagram tasks.

\section{Reproduction of the Asch Experiments Without Using Confederates}

Asch (1956) showed that a minority of one tended to conform to a unanimous majority even when the majority seemed to have made a wrong choice on a simple visual discrimination task. In the Asch experiments, the majority comprised confederates and only the minority participants were naive. However, if it had been possible to present two different sets of lines to participants, the Asch experiments could have been done without using confederates. Mori and Arai (2007) tried to replicate the experimental condition without using confederates by utilizing the fMORI technique.

The nine stimulus sets used in Asch (1956) were reproduced in approximately the same size when projected on the screen using digital picture editing software (Adobe Photoshop). The top parts of the standard lines were drawn in either green or magenta so that two groups of participants could be shown them differently to form a majority and a minority utilizing the fMORI technique (see Figure 5).

The stimuli were presented by means of PowerPoint slides with a personal computer (Apple iBook) and projected by an LCD projector (EPSON ELP-730) onto a rear screen made from an office partition $183 \mathrm{~cm}$ in height and $146 \mathrm{~cm}$ in width in which the window glass pane had been changed from patterned to plain ground glass $(80 \times$ $142 \mathrm{~cm}$ ). The rear screen was set about $2 \mathrm{~m}$ away from the projector. Four chairs were placed in line side by side about $2 \mathrm{~m}$ apart on the other side of the screen.

Participants were told to wear a pair of polarizing sunglasses to protect their eyes from glare. Three pairs were used to form the majority and one pair was used for the minority. The sunglasses were placed on the chairs before the participants entered the laboratory. The minority participants' sunglasses were placed on the third chair.

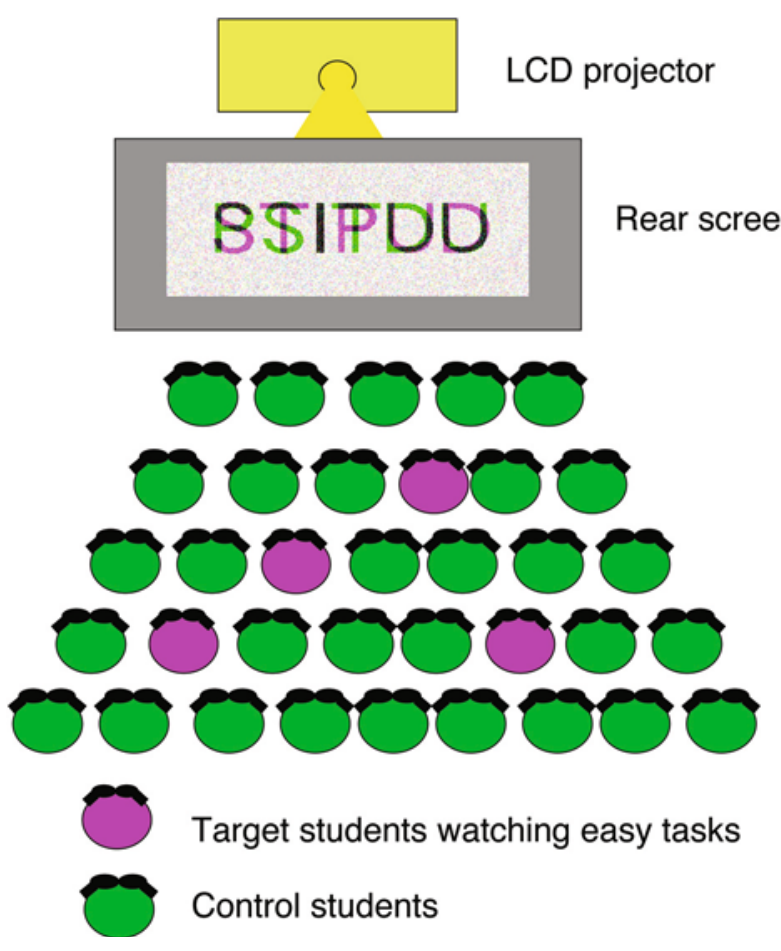

Figure 4. The experimental setting for Mori and Uchida (2007). 


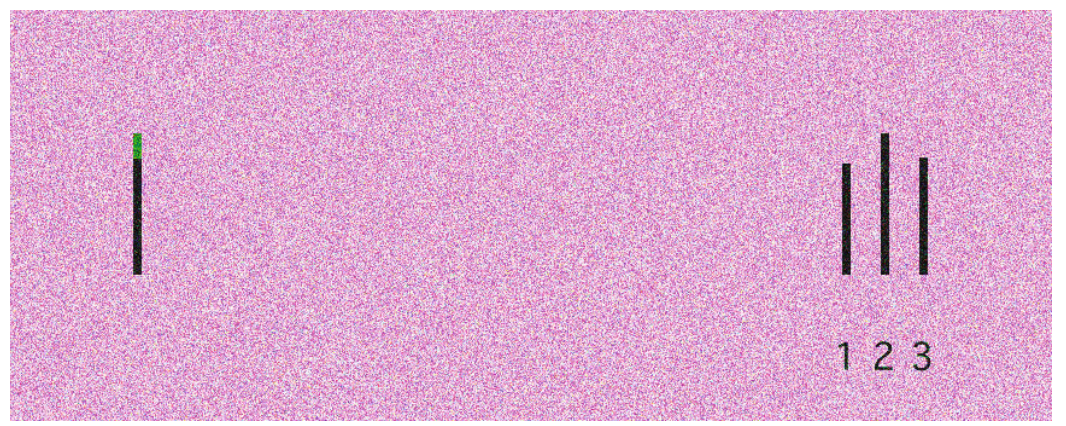

Figure 5. An example of standard and comparisons (Mori \& Arai, 2007). The greenish part on the top of the standard can or cannot be seen depending on the type of polarizing sunglasses worn by the participant. The background is colored appropriately to maximize the separation of the two images. Random dot noises are added for the same purpose.

Twenty-six same-sex groups of 4 (10 groups of males and 16 groups of females) participated in the study by Mori and Arai (2007), and none of these participants noticed the duality. Three participants in each group unanimously produced answers different from that of the lone participant. The female minority participants showed a conforming tendency similar to that found by Asch (1956): 28.6\% of their responses were conformity errors, in comparison with $36.8 \%$ for Asch's male participants. However, the male minority participants made far fewer conforming responses $(5.0 \%)$. Despite these minor differences, the most important point is that Asch's experiment was successfully reproduced without the use of confederates.

\section{CONCLUSION}

The fMORI technique introduced in this paper is a variation of the MORI technique utilizing the same basic principle: the polarization of light. Both techniques can present two different visual stimuli surreptitiously to two groups of viewers. Although the fMORI technique has some limitations in that it can present stimuli only in monochrome and it cannot present movies, it is far easier to set up because it requires only one projector.

This setup simplicity makes it far more useful for psychological research equipment than the original MORI technique because some areas of experimental research in psychology use only simple written verbal materials, such as words or sentences, or other static monochrome stimuli, which can be easily presented by means of the fMORI technique. Thus far, the MORI technique has only been applied to a limited research topic such as eyewitness memory experiments. However, the fMORI technique can be used in research on social comparison (Mori, 2007a), on self-efficacy promotion (Mori \& Uchida, 2007), and on conformity of the minority (Mori \& Arai, 2007) as briefly described above. These sample applications may evoke other research ideas to which it can be applied by the readers of this article.

\section{AUTHOR NOTE}

This research was supported by Grant-in-Aid 16653054 from the Japanese Ministry of Education, Culture, Sports, Science, and Technology. I express thanks to my students, Akitoshi Uchida, Yuki Miyagawa, and Miho Arai, who contributed to the improvement of the technique and conducted experiments using it. I also thank Rebecca Ann Marck for her assistance in the preparation of the English manuscript. Correspondence concerning this article should be addressed to K. Mori, who is now at Tokyo University of Agriculture and Technology, Koganei, Tokyo, Japan (e-mail: kaz-mori@cc.tuat.ac.jp).

\section{REFERENCES}

Alicke, M. D., LoSchiavo, F. M., Zerbst, J., \& Zhang, S. (1997). The person who outperforms me is a genius: Maintaining perceived competence in upward social comparison. Journal of Personality \& Social Psychology, 73, 781-789.

Asch, S. E. (1956). Studies of independence and conformity: I. A minority of one against a unanimous majority. Psychological Monograph: General \& Applied, $\mathbf{7 0}(9$, Whole No. 416).

Garry, M., French, L., Kinzett, T., \& Mori, K. (in press). Eyewitness memory following discussion: Using the MORI technique with a western sample. Applied Cognitive Psychology.

Kanematsu, H., Mori, K., \& Mori, H. (2003). Memory distortion in eyewitness pairs who observed nonconforming events and discussed them. Journal of the Faculty of Education, Shinshu University, 109, 75-84. (Original work published in Japanese in 1996)

MoRI, K. (2003). Surreptitiously projecting different movies to two subsets of viewers. Behavior Research Methods, Instruments, \& Computers, 35, 599-604.

Mori, K. (2007a). No "genius effect" among friends: Social comparison without using confederates. Manuscript submitted for publication.

MoRI, K. (2007b). A revised method for projecting two different movies to two groups of viewers without their noticing the duality. Behavior Research Methods, 39, 574-578.

Mori, K., \& AraI, M. (2007, May). Replication of the Asch experiment without using confederates. Poster presented at the 19th Annual Convention of the Association for Psychological Science, Washington, D.C.

MORI, K., \& UCHIDA, A. (2007). Can artificially contrived success affect the self-efficacy among junior high school students? Manuscript submitted for publication.

(Manuscript received October 13, 2006; revision accepted for publication January 23, 2007.) 